\title{
CORRELAÇÃO FENOTÍPICA ENTRE CARACTERES DE HÍBRIDOS DIPLOIDES (AA) DE BANANEIRA ${ }^{1}$
}

\author{
LAURO SARAIVA LESSA ${ }^{2}$, CARLOS ALBERTO DA SILVA LEDO 3 , \\ EDSON PERITO AMORIM ${ }^{3}$, SEBASTIÃO DE OLIVEIRA E SILVA ${ }^{3}$
}

RESUMO - O estudo de correlação tem como propósito mensurar a alteração em um caráter quando se altera outro. Neste trabalho, objetivou-se estimar correlações fenotípicas entre o número de frutos por cacho e 22 caracteres avaliados em híbridos diploides (AA) de bananeira. No experimento, conduzido na Embrapa Mandioca e Fruticultura, em blocos casualizados com quatro repetições, foram avaliados 11 híbridos diploides (AA) de bananeira. Os caracteres avaliados foram: altura de plantas, diâmetro do pseudocaule, número de filhos, número de folhas na floração, período do plantio ao florescimento, presença de pólen, peso do cacho e da ráquis, sigatoka-amarela no florescimento, número de folhas na colheita, Sigatoka-amarela na colheita do cacho, número de dias do florescimento à colheita, comprimento e diâmetro do engaço, peso da segunda penca, número de pencas e de frutos por cacho, fragilidade do pedicelo, comprimento e diâmetro do fruto e comprimento do pedicelo, além de presença de semente. Após a tabulação, procederam-se a estudos de correlação entre o número de frutos e os demais caracteres da planta. Essas correlações variaram entre os genótipos, sendo assim, observado que as associações entre o número de frutos e os caracteres vegetativos da planta foram, de forma geral, não significativas. Já as relações entre o número de frutos por cacho e os outros caracteres produtivos foram, predominantemente, significativas.

Termos para indexação - Musa acuminata, variabilidade, melhoramento.

\section{PHENOTYPIC CORRELATION BETWEEN CHARACTERS IN BANANA (AA) DIPLOID HYBRIDS}

\begin{abstract}
The objective of the present study was to estimate the phenotypic correlations between the number of fruits per bunch and 22 characters evaluated in banana (AA) diploid hybrids. The experiment was carried out at Embrapa Cassava and Fruits in randomized blocks with four repetitions and 11 (AA) banana diploid hybrids were evaluated. The following characteristics were evaluated: plant height, pseudostem diameter, number of suckers, number of leaves during flowering, plant cycle until emission of the bunch, presence of pollen, bunch and rachis weight, yellow Sigatoka at bunch emission, number of leaves at harvest, yellow Sigatoka at bunch harvest, number of days from emission to harvest, length and diameter of stem, weight of second hand, number of hands and fruits per bunch, pedicellum fragility, length and diameter of fruit and length of pedicellum and presence of seeds. Correlation studies between the number of fruits and the other plant characteristics were carried out. These correlations varied between genotypes; therefore, it was observed that the associations between number of fruits and vegetative characteristics of plants were, in general, non-significant. However, the relationships between the number of fruits per bunch and the other production characteristics were predominantly significant.
\end{abstract}

Index terms: Musa acuminata, variability, breeding.

\footnotetext{
${ }^{1}$ (Trabalho 081-12). Recebido em: 31-01-2012. Aceito para publicação em: 25-07-2012. Parte de Dissertação de Mestrado do primeiro autor.

${ }^{2}$ Eng $^{\circ}$ Agr ${ }^{\circ}$. M.Sc. Embrapa Acre. BR 364, km 14, CP 321, CEP 69908-970, Rio Branco - AC. E-mail: lauro.lessa@embrapa.br

${ }^{3} \mathrm{Eng}^{\circ} \mathrm{Agr}^{\circ}$. D.Sc. Embrapa Mandioca e Fruticultura Tropical. Rua da Embrapa s/n. C. P.: 007. CEP 44.380-000, Cruz das Almas - BA. E-mails:carlos.ledo@embrapa.br, edson.amorim@cnpmf.embrapa.br, sebastião.silva@colaborador.embrapa.br
} 


\section{INTRODUÇÃO}

A falta de cultivares de bananeira que apresentem características agronômicas superiores, constitui-se em um fator limitante para a cultura. A estratégia mais utilizada para a solução deste problema está no desenvolvimento de novos genótipos, obtidos em programas de melhoramento genético, e sua avaliação e caracterização em áreas de produção (SILVA et al., 2000). Para se obter esses genótipos, o requisito básico é explorar a variabilidade disponível entre os diploides (AA) de bananeira (SHEPHERD, 1992; DANTAS et al., 1999; SILVA et al., 2002).

O maior conhecimento dos diploides (AA) e da associação entre seus caracteres, pode ser feito por meio do estudo das correlações, que são relações entre fatores genéticos e ambientais, que têm como propósito mensurar a alteração em um caráter quando se altera outro. Ao determinar a magnitude e a significância das associações entre descritores fenotípicos, utilizado para a seleção de indivíduos em trabalhos de avaliação, pode-se discriminar quais influenciam na produção (VENCOVSKY e BARRIGA, 1992).

Atributos morfológicos que exerçam efeitos na produção podem ser definidos por meio das correlações entre caracteres do desenvolvimento vegetativo e caracteres do cacho (SIQUEIRA, 1984). Desta forma, foi observado que a produtividade em bananeira (peso de cacho por área) é função da quantidade de frutos e do peso médio dos frutos da planta. O número de frutos está estreitamente relacionado com o número de pencas (TURNER, 1980; JARAMILLO, 1982; CARVALHO, 1995; DONATO, 2006)

Assim, este trabalho objetivou estimar correlações entre o número de frutos por cacho e outros caracteres da planta, observados do florescimento à colheita, em híbridos diploides (AA) de bananeira.

\section{MATERIAL E MÉTODOS}

O experimento foi instalado em um Latossolo Amarelo distrófico e conduzido no Campo Experimental da Embrapa Mandioca e Fruticultura, em Cruz das Almas - BA, situada a 12 40 ' 19 " de Latitude sul e $39^{\circ} 06^{\prime} 22^{\prime \prime}$ de Longitude oeste, tendo $220 \mathrm{~m}$ de altitude. O clima é tropical quente e úmido, Aw a Am, segundo a classificação de Köppen, com temperaturas médias anuais de $24,5^{\circ} \mathrm{C}$ e umidade relativa de $80 \%$.

Foram avaliados em um ciclo de produção 11 híbridos diploides (AA) de bananeira (04207906; TH03-01; 089087-01; 003023-03; 013018-01; 001016-01;086094-20;013004-06; 091079-03; 042023-06; SH32-63), dispostos em blocos casualiza- dos com quatro repetições. Cada parcela constituiu-se de seis plantas, espaçadas $2,5 \mathrm{~m} \times 2,5 \mathrm{~m}$,tendo como bordadura externa a cultivar Pacovan.

Avaliaram-se os seguintes caracteres: altura de plantas (ALT - m); diâmetro do pseudocaule (DPC - cm); número de filhos na floração (NFI); número de folhas na floração (NFF); número de dias do plantio ao florescimento (CPE - dias); presença de pólen (POL); peso do cacho (PCA - kg); peso da ráquis $(\mathrm{PRQ}-\mathrm{g})$; Sigatoka-amarela no florescimento (SAE) e na colheita (SAC), segundo a escala de notas de Stover (1972); número de folhas na colheita (NFC); número de dias do florescimento à colheita (CEC - dias); comprimento (CEG - $\mathrm{cm})$ e diâmetro do engaço (DEN $-\mathrm{cm}$ ); peso da segunda penca (PSP - g); número de pencas por cacho (NPE); fragilidade do pedicelo (FPE), de acordo com a seguinte escala de notas: 1 - frágil; 2 - pouco frágil, e 3 - não frágil; comprimento $(\mathrm{CMF}-\mathrm{cm})$ e diâmetro do fruto $(\mathrm{DMF}-\mathrm{cm})$; comprimento $(\mathrm{CMP}-\mathrm{mm})$ e diâmetro do pedicelo (DMP $-\mathrm{mm}$ ), e presença de sementes (SEM), de acordo com a seguinte escala: 1 - ausente $(\mathrm{SEM}=0) ; 2$ - pouco $(1 \leq \mathrm{SEM} \leq 10) ; 3$ - médio ( $11 \leq \mathrm{SEM} \leq 20) ; 4-$ abundante $(\mathrm{SEM} \geq 21)$.

Para cada genótipo, foram estimadas as correlações lineares, referentes às associações entre 0 número de frutos (NFR) e os 21 demais caracteres, utilizando-se do programa estatístico SAS versão 9.1 (SAS INSTITUTE INC. 2002 - 2003).

\section{RESULTADOS E DISCUSSÃO}

A associação entre o número de frutos por cacho e a altura das plantas para a maioria dos genótipos foi não significativa, exceto para os híbridos, 089087-01 e 042023-06 (Tabela 2), podendo existir dependência linear entre as características para os referidos diploides. Resultados semelhantes foram observados por Lima Neto et al. (2003) em genótipos triploides e tetraploides de bananeira.

A relação entre o diâmetro do pseudocaule, o número de filhos e de folhas na floração, com o número de frutos, foi predominantemente não significativa para a maioria dos diploides, exceto para os genótipos 003023-03 e 091079-03, que apresentaram correlações significativas para estas associações (Tabela 2). Estudos de correlação entre os caracteres vegetativos, em alguns trabalhos (HOLDER; CUMBS, 1982; SIQUEIRA 1984; FERNANDEZ CALDAS et al.,1977), demonstraram que o diâmetro do pseudocaule, seguido do número de folhas no florescimento foram as características que mais se correlacionaram com o caráter produção.

A associação entre o número de frutos e o nú- 
mero de dias do plantio ao florescimento apresentou, para todos os genótipos, estimativas de correlação não significativas (Tabela 2). Com exceção dos genótipos TH03-01, 013018-01 e SH32-63, a associação entre o número de frutos e a presença de pólen foi, predominantemente, não significativa (Tabela 2).

Para a associação entre o número de frutos e o peso do cacho, à exceção dos híbridos 089087-01 e 086094-20, que apresentaram estimativas não significativas, e do híbrido 013004-06, que apresentou correlação linear negativa, os demais genótipos apresentaram estimativas positivas e de alta magnitude, confirmando os resultados observados por Lima Neto et al. (2003), em cultivares de bananeira. Tal resultado já é esperado, uma vez que a dependência linear entre essas características é muito acentuada. $\mathrm{Na}$ associação entre o número de frutos por cacho e o peso da ráquis, as estimativas foram, predominantemente, não significativas, como pode ser visto na Tabela 2. Estes dados estão de acordo com os observados por Donato et al. (2006) para a associação entre essas duas características.

A relação número de frutos por cacho e a nota de Sigatoka-amarela no florescimento mostrou-se não significativa nos genótipos TH03-01, 089087-01, 003023-03, 013004-06, 091079-03 e 042023-06, o que pode ser atribuído a uma resistência parcial dos genótipos ao patógeno, uma vez que, nesta fase, a planta se encontra fragilizada, com suas reservas nutricionais particionadas e demandadas para a formação do cacho. A mesma relação apresentou significância, de forma direta, para os genótipos 042079-06, 013018-01, 086094-20 e SH32-63 (Tabela 2). Para estes genótipos, a presença do patógeno, durante seu ciclo vegetativo, não afetou a formação dos frutos por cacho, dada a baixa virulência da doença. No entanto, para o genótipo 001016-01, esta associação mostrou-se significativa e negativa, indicando que este híbrido pode ser suscetível à doença.

Quanto à associação entre número de frutos por cacho e número de folhas vivas na colheita, observaram-se relações, predominantemente, não significativas para os genótipos avaliados, exceto para os híbridos 013018-01, 091079-03 e SH3263, que apresentaram relação linear, significativa e positiva, para essa associação, indicando que, para estes genótipos, quanto maior o número de folhas na colheita, maior será o número de frutos contidos nos respectivos cachos. Segundo Soto Ballestero (1992), as plantas de bananeira tipo Cavendish necessitam, no mínimo, de oito folhas para a boa formação do cacho.

Nos genótipos 042079-06, 003023-03, 013018-01 e 086094-20, observou-se relação linear significativa e negativa, de alta magnitude, para a associação entre número de frutos por cacho e a presença de Sigatoka-amarela na colheita (Tabela 2), indicando que, para estes híbridos, a presença do fungo influenciou negativamente a formação dos cachos. Já o genótipo SH32-63 apresentou relação linear significativa e positiva para esta associação, implicando que a presença do fungo não influenciou no número de frutos formados.

Apesar de a Sigatoka-amarela afetar a eficiência da folha em realizar a fotossíntese, a produção de frutos depende não só deste fator, mas principalmente do número de folhas na planta. Lima Neto et al. (2005) afirmam que o enchimento do fruto está diretamente correlacionado ao número de folhas na colheita.

As associações entre número de frutos por cacho e número de dias do florescimento à colheita foram, em sua maioria, negativas e não significativas. Correlações significativas e negativas, de alta magnitude, ocorreram nos genótipos 013018-01, 1304-06 e SH32-63, confirmando os resultados de Donato et al. (2006) para esta relação.

Observou-se a predominância de estimativas não significativas na relação do comprimento de engaço com o número de frutos. Por outro lado, estimativas significativas, negativas e de alta magnitude foram observadas na associação do número de frutos com o diâmetro do engaço (Tabela 3). Resultados semelhantes para essas correlações foram observados por Fernandez Caldas et al. (1977), Lima Neto et al. (2003) e Donato et al. (2006).

A associação entre número de frutos e peso da segunda penca foi, predominantemente, significativa, negativa e de alta magnitude para a maioria dos genótipos. Estimativas não significativas, para esta relação, foram observadas nos genótipos TH03-01, 013004-06, 042023-06 e SH32-63.

Semelhantemente aos resultados observados em vários trabalhos (LOSSOIS, 1963; HOLDER ; CUMBS, 1982; LIMANETO et al., 2003; DONATO et al., 2006), a associação entre o número de frutos por cacho e o número de pencas apresentou relação linear, positiva e de alta magnitude para a maioria dos genótipos (Tabela 3), o que já é esperado, uma vez que a dependência linear entre as características é muito alta. Por outro lado, o híbrido 013004-06 apresentou correlação linear negativa para esta associação, fato este que pode estar relacionado à natureza genética da planta.

A relação entre o número de frutos por cacho e a fragilidade do pedicelo apresentou predominância de estimativas positivas e altas. O híbrido 013004-06 apresentou correlação negativa e significativa para esta associação, e os genótipos TH03-01, 089087-01 
e 042023-06 apresentaram estimativas não significativas para a mesma.

As estimativas de correlação linear entre o número de frutos por cacho e o comprimento do fruto foram, predominantemente, positivas e de alta magnitude para a maioria dos genótipos. Já a relação entre o número de frutos e o diâmetro do fruto mostrou-se significativa, porém negativa para a maioria dos genótipos (Tabela 3). Estas associações, embora assumam valores, predominantemente, positivos para comprimento e negativo para diâmetro, denotam a associação genética entre os caracteres mencionados. Resultados semelhantes foram obtidos por Pádua (1978) e Siqueira (1984), trabalhando com cultivares de bananeira.
O comprimento do pedicelo apresentou menor relação com o número de frutos por cacho que o diâmetro do pedicelo. Isso pode ser observado nas estimativas de correlações para ambos os caracteres, em que, no comprimento do pedicelo, apresentou predominância de valores não significativos, e o diâmetro, de estimativas significativa e negativa na relação com o número de frutos (Tabela 3 ).

Os genótipos 042079-06, 001016-01, 08609420 e SH32-63, apresentaram estimativas significativas para a associação entre número de frutos por cacho e presença de sementes. Entretanto, o híbrido 00101601 apresentou valor negativo para esta associação. Os demais genótipos apresentaram estimativas não significativas para esta relação, como pode ser observado na Tabela 3.

TABELA 1 - Códigos dos híbridos diploides (AA) utilizados no experimento e seus respectivos genótipos parentais.

\begin{tabular}{lll}
\hline $\begin{array}{c}\text { Híbridos } \\
\text { Diploides** }\end{array}$ & $\begin{array}{c}\text { Grupo } \\
\text { Genômico }\end{array}$ & \multicolumn{1}{c}{ Parentais } \\
\hline $042079-06$ & AA & - M53 x 2803 (Tuu Gia x Calcutta) \\
TH03-01 & AA & - Terrinha x Calcutta \\
$089087-01$ & AA & - 013018-01 (Malaccensis FHIA x Sinwobogi) x 003038-01(Calcutta x Heva) \\
$003023-03$ & AA & - Calcutta (M. acuminata spp. burmannica) x Cultivar sem nome \\
$013018-01$ & AA & - Malaccensis FHIA x Sinwobogi \\
$001016-01$ & AA & - Borneo x Guyod \\
$086094-20$ & AA & - 003037-02 (Calcutta x Galeo) x SH32-63 \\
$013004-06$ & AA & - Malaccensis x Madang (Musa acuminata spp. banksii) \\
$091079-03$ & AA & - 001016-01 (Borneo x Guyod) x 028003 (Tuu Gia x Calcutta) \\
$042023-06$ & AA & - M53 x Cultivar sem nome ${ }^{\circ} 2$ \\
SH32-63 & AA & - Híbrido selecionado em Honduras \\
\hline
\end{tabular}

** Os dois primeiros números referem-se ao genitor feminino; os dois seguintes, ao genitor masculino, e os dois últimos, ao número da seleção.

TABELA 2-Correlações fenotípicas entre o número de frutos e altura de plantas (ALT), diâmetro do pseudocaule (DPC), número de filhos na floração (NFI), número de folhas na floração (NFF), número de dias do plantio à floração $(\mathrm{CPE})$, presença de pólen $(\mathrm{POL})$, peso do cacho $(\mathrm{PCA})$, peso da ráquis (PRQ), Sigatoka-amarela no florescimento (SAE), número de folhas na colheita (NFC) e Sigatoka-amarela na colheita do cacho (SAC), em híbridos diploides (AA) de bananeira.

\begin{tabular}{|c|c|c|c|c|c|c|c|c|c|c|c|}
\hline \multirow[b]{2}{*}{ Genótipos $^{1}$} & \multicolumn{11}{|c|}{ Correlação entre o número de frutos por cacho e os caracteres } \\
\hline & ALT & $\mathrm{DPC}$ & NFIF & NFF & $\mathrm{CPE}$ & POL & PCA & PRQ & SAE & $\mathrm{NFC}$ & $\mathrm{SAC}$ \\
\hline $042079-06$ & & & $-0,321^{\mathrm{ns}}$ & & & & & -0, & & & \\
\hline & & $36^{\mathrm{ns}}$ & ns & -( & 1 & $803 * *$ & * & 0,44 & & $5^{\text {ns }}$ & 1 ns \\
\hline & & ns & $-0,113^{\mathrm{ns}}$ & & $0,473^{\mathrm{ns}}$ & & & ns & & $2^{\text {ns }}$ & \\
\hline & & & ns & & & NS & $* *$ & -0 , & & & \\
\hline & & $2^{\text {ns }}$ & 0,29 & -( & 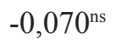 & $*$ & 006 & -0 & 0 , & $0,727^{*}$ & $-0,625 * *$ \\
\hline & & $1^{\mathrm{ns}}$ & 0,1 & & $0, \mathrm{C}$ & ns & 0,98 & $-0,56$ & -0, & $0,411^{\mathrm{ns}}$ & $-0,035^{\mathrm{ns}}$ \\
\hline & $4^{\mathrm{ns}}$ & $0,467^{\mathrm{ns}}$ & $-0,206^{\mathrm{ns}}$ & -0 & $-0,1$ & $98^{\mathrm{ns}}$ & $0,391^{\mathrm{ns}}$ & $-0,603^{* *}$ & $0,798 * *$ & $0,184^{\mathrm{ns}}$ & $-0,817 * *$ \\
\hline & $79^{\mathrm{ns}}$ & $0,195^{\mathrm{ns}}$ & $-0,071^{\mathrm{ns}}$ & 0,0 & $-0,210^{\mathrm{ns}}$ & $-0,841 * *$ & $-0,807 * *$ & $0,747 * *$ & $0,479^{\text {ns }}$ & $-0,824 * *$ & $-0,363^{\mathrm{ns}}$ \\
\hline & & & & $-0,0$ & $-0,12$ & & $0,992 * *$ & $-0,732^{*}$ & & $0,840 * *$ & $-0,218^{\mathrm{ns}}$ \\
\hline & & & $0,706^{\mathrm{ns}}$ & & 0,61 & & & $0,287^{\mathrm{ns}}$ & & & $0,193^{\mathrm{ns}}$ \\
\hline $\mathrm{SH} 32-63^{2}$ & $0,126^{\mathrm{ns}}$ & $0,201^{\mathrm{ns}}$ & $-0,394^{\mathrm{ns}}$ & $0,130^{\text {ns }}$ & $-0,370^{\mathrm{ns}}$ & $0,999 * *$ & $0,999 * *$ & $-0,763^{\mathrm{ns}}$ & $0,787 * *$ & $0,999 * *$ & $0,787 * *$ \\
\hline
\end{tabular}

\footnotetext{
** e *, significativos a $1 \%$ e a $5 \%$ de probabilidade, respectivamente, pelo teste $\mathrm{t}$; ${ }^{\text {ns }}$ não significativo.

${ }^{1}$ Os dois primeiros números referem-se ao genitor feminino; os outros dois, ao genitor masculino, e os dois últimos, ao número da seleção.

${ }^{2}$ Híbrido selecionado em Honduras.
} 
TABELA 3- Correlação fenotípica entre o número de frutos e o número de dias do florescimento à colheita do cacho (CEC), o comprimento do engaço (CEG), o diâmetro do engaço (DEN), o peso da segunda penca (PSP), o número de pencas por cacho (NPE), a fragilidade do pedicelo (FPE), o comprimento do fruto (CMF), o diâmetro do fruto (DMF), o comprimento do pedicelo (CMP), o diâmetro do pedicelo (DMP) e a presença de sementes (SEM), em híbridos diploides de bananeira.

\begin{tabular}{|c|c|c|c|c|c|c|c|c|c|c|c|}
\hline \multirow[b]{2}{*}{ Genótipos $^{1}$} & \multicolumn{11}{|c|}{ Correlação entre o número de frutos por cacho e os caracteres } \\
\hline & $1 \mathrm{CEC}$ & CEG & DEN & PSP & NPE & FPE & $\mathrm{CMF}$ & DMF & CMP & DMP & SEM \\
\hline $042079-06$ & $0,527^{\mathrm{ns}}$ &, $704 * *$ & $-0,965 * *$ & $-0,823 * *$ &, $999 * *$ & $0,988 * *$ & $0,946^{* *}$ & $-0,879 * *$ & $0,4382^{\mathrm{ns}}$ & $-0,894 * *$ & $0,921 * *$ \\
\hline & $0,210^{\mathrm{ns}}$ & $-0,228^{\mathrm{ns}}$ & $311^{\mathrm{ns}}$ & $336^{\mathrm{ns}}$ & $0,740 * *$ & $0,015^{\mathrm{ns}}$ & $0,143^{\mathrm{ns}}$ & $0,085^{\mathrm{ns}}$ & $-0,403^{\mathrm{ns}}$ & $-0,188^{\mathrm{ns}}$ & $0,220^{\mathrm{ns}}$ \\
\hline & $-0,481^{\mathrm{ns}}$ & $0,049^{\text {ns }}$ & $0,191^{\mathrm{ns}}$ & $*$ & 0,62 & $-0,076^{\mathrm{ns}}$ & $-0,247^{\mathrm{ns}}$ & -0 & $0,200^{\mathrm{ns}}$ & ns & $0,056^{\mathrm{ns}}$ \\
\hline 03 & $0,150^{\mathrm{ns}}$ & $0,406^{\mathrm{ns}}$ & $-0,918 * *$ & $-0,706 * *$ & $0,991 * *$ & $0,945 * *$ & $0,748 * *$ & $3 * *$ & $-0,319^{\mathrm{ns}}$ & $-0,815^{* *}$ & $0,378^{\text {ns }}$ \\
\hline 01 & $-0,840 * *$ & $0,132^{\mathrm{ns}}$ & $-0,867 * *$ & $-0,613 * *$ & $0,901 * *$ & $0,975 * *$ & 0,7 & -0 & $21^{\mathrm{ns}}$ & -0 & $-0,512^{\mathrm{ns}}$ \\
\hline & $0,157^{\mathrm{ns}}$ & $0,487 *$ & $-0,892 * *$ & $-0,518^{*}$ & $0,984 * *$ & $0,972 * *$ & 0 & & $-0,0$ & $-0,7$ & $-0,747 * *$ \\
\hline & $-0,419^{\text {ns }}$ & $0,034^{\mathrm{ns}}$ & $-0,862 * *$ & $-0,687 * *$ & 0,864 & $0,843 * *$ & 0,7 & -0 , & -0 , & $-0,785^{* *}$ & $0,589 * *$ \\
\hline & $0,793 * *$ & $0,698 * *$ & $-0,782 * *$ & $0,467^{\mathrm{ns}}$ & $-0,824 * *$ & $-0,644^{*}$ & $0,421^{\mathrm{ns}}$ & $9 *$ & $0,411^{\mathrm{ns}}$ & $0,636^{*}$ & $0,115^{\mathrm{ns}}$ \\
\hline 03 & $-0,133^{\mathrm{ns}}$ & $-0,246^{\mathrm{ns}}$ & $-0,911 * *$ & $-0,707 * *$ & $0,97686 * *$ & $0,778^{*} *$ & $0,692 * *$ & $-0,631 *$ & $-0,576^{*}$ & $-0,705^{* *}$ & $-0,527^{\mathrm{ns}}$ \\
\hline & $-0,442^{\mathrm{ns}}$ & $-0,124^{\mathrm{ns}}$ & $-0,642^{\mathrm{ns}}$ & $0,525^{\mathrm{ns}}$ & $0,794 *$ & $-0,563^{\mathrm{ns}}$ & $-0,495^{\mathrm{ns}}$ & $-0,630^{\mathrm{ns}}$ & $-0,029^{\mathrm{ns}}$ & $-0,518^{\mathrm{ns}}$ & $0,559^{\text {ns }}$ \\
\hline SH32-632 & $-0,997 * *$ & $0,548^{\mathrm{ns}}$ & $-0,913 *$ & $0,163^{\mathrm{ns}}$ & $0,999 * *$ & $0,998^{*}$ & $0,983 * *$ & $-0,822^{\mathrm{ns}}$ & $-0,939 *$ & $-0,993 * *$ & $0,925^{*}$ \\
\hline
\end{tabular}

** e *, significativos a $1 \%$ e a $5 \%$ de probabilidade, respectivamente, pelo teste t; ${ }^{\text {ns }}$ não significativo.

${ }^{1}$ os dois primeiros números referem-se ao genitor feminino; os outros dois, ao genitor masculino, e os dois últimos, ao número da seleção.

${ }^{2}$ Híbrido selecionado em Honduras.

\section{CONCLUSÕES}

1-As correlações entre o número de frutos e os demais caracteres estudados variam entre os híbridos estudados.

2-As associações entre o número de frutos e os caracteres vegetativos da planta, são, de forma geral, não significativas.

3-As relações entre o número de frutos por cacho e os caracteres produtivos e do cacho são, predominantemente, significativas, indicando dependência linear entre as referidas características no ambiente de Cruz das Almas-BA.

\section{AGRADECIMENTOS}

À Embrapa Mandioca e Fruticultura, pela concessão de material vegetal e área para a realização do referido estudo. À Capes, pela concessão da bolsa, e à UFRB, pela oportunidade de realização do treinamento de Mestrado.

\section{REFERÊNCIAS}

CARVALHO, P.C.L. de. Estabelecimento de descritores botânicos-agronômicos para caracterização de banana (Musa spp). 1995. 190 f. Dissertação (Mestrado em Fruticultura Tropical) - Escola de Agronomia, Universidade Federal da Bahia, Cruz das Almas, 1995.
DANTAS, J.L.L. et al. Classificação botânica, origem e distribuição geográfica. In: ALVES, E.J. (Org.). A cultura da banana: aspectos técnicos, sócioeconômicos e agrindustriais. 2 ed. Cruz das Almas: Embrapa CNPMF, 1999. p. 27-34.

DONATO, S.L.R. et al. Correlação entre caracteres da planta e do cacho em bananeira (Musa spp). Ciência e Agrotecnologia, Lavras, v. 30, n. 1, p. 21-30, 2006.

FERNANDES CALDAS, E. et al. Análisis foliar del plátano em dos fases de su floración. Fruits, Paris, v. 32, n. 9, p. 227-251, 1977.

HOLDER, G.D.; CUMBS, F.A. Effects of water supply during floral initiation and differentiation on female flower production by Robusta banana. Experimental Agriculture, New York, v. 18, n. 2, p. 109-117, 1982.

JARAMILLO, R.C. Las principales características morfológicas del fruto de banano, variedade Cavendish Gigante (Musa AAA) en Costa Rica. Costa Rica: Upeb - Impretex, 1982. 42 p.

LIMA NETO, F.P. et al. Relação entre caracteres de rendimento e desenvolvimento em genótipo de bananeira. Magistra, Cruz das Almas, v. 15, n. 2, p.275-281, 2003. 
LOSSOIS, P. Recherche d'une méthode de prévision dês récoltes em culture bananière. Fruits, Paris, v. 18, n. 6, p. 283-293, 1963.

PÁDUA, T. Caracterização agronômica do cacho da bananeira 'Prata'. 1978. 117 f. Dissertação (Mestrado em Agronomia) - Escola Superior de Agricultura de Lavras, Lavras, 1978.

SAS INSTITUTE. Statistical analysis system: release 9.1. Cary, 2002-2003.

SHEPHERD, K. et al. Melhoramento genético da bananeira. Informe Agropecuário, Belo Horizonte, v.12 p.11-19, 1992.

SILVA, S.O. et al. Avaliação de cultivares de bananeira em quatro ciclos de produção. Pesquisa Agropecuária Brasileira, Brasília, v. 37, n. 11, p. $1567-1574,2002$.

SILVA, S.O. et al. Caracterização morfológica e avaliação de cultivares e híbridos de bananeira. Revista Brasileira de Fruticultura, Jaboticabal, v. 22, n. 2 , p. 161-169, 2000.
SIQUEIRA, D.L. Variabilidade e correlação de caracteres em clones da bananeira 'Prata'. 1984. 66 f. Dissertação (Mestrado em Agronomia) - Escola Superior de Agricultura de Lavras, Lavras, 1984.

SOTO BALLESTERO, M. Bananos: cultivo y comercialización. 2. ed. San

STOVER, R.H. Banana, plantain and abaca disease. Washington: Commonwealth Mycological Institute, 1972. 318p.

TURNER, D.W. Some factors related to yield components of bananas, in relation to sampling to assess nutrient status. Fruits, Paris, v. 35, n. 1, p. $19-23,1980$.

VENCOVSKY, R.; BARRIGA, P. Associação entre caracteres. In: VENCOVSKY, R.; BARRIGA, P. Genética biométrica no fitomelhoramento. Ribeirão Preto: Sociedade Brasileira de Genética, Ribeirão Preto, 1992. p. $335-434$. 